\title{
Erratum: Search for ultralight bosons in Cygnus X-1 with Advanced LIGO [Phys. Rev. D 101, 063020 (2020)]
}

\author{
Ling Sun®, ${ }^{1,2, *}$ Richard Brito, ${ }^{3}$ and Maximiliano $\mathrm{Isi}^{4}$ \\ ${ }^{1} \mathrm{OzGrav}-\mathrm{ANU}$, Centre for Gravitational Astrophysics, College of Science, The Australian National \\ University, ACT 2601, Australia \\ ${ }^{2}$ LIGO Laboratory, California Institute of Technology, Pasadena, California 91125, USA \\ ${ }^{3}$ Dipartimento di Fisica, "Sapienza" Universita di Roma \& Sezione INFN Roma1, Piazzale Aldo Moro 5, \\ 00185, Roma, Italy \\ ${ }^{4}$ LIGO Laboratory, Massachusetts Institute of Technology, Cambridge, Massachusetts 02139, USA
}

(Received 15 July 2020; published 26 October 2020)

DOI: 10.1103/PhysRevD.102.089902

This erratum reports a missing coefficient when comparing the numerically estimated signal strain $h_{0}$ to the upper limit $h_{0}^{95 \%}$ in Sec. III A of this paper. The numerically estimated signal strain defined in (28) in Ref. [1] differs from the conventional definition of $h_{0}$ in continuous-wave literature by a factor of $\sqrt{5 /(4 \pi)}$ [2] .

This missing factor has been corrected in the original Fig. 3 in this paper, as shown in Fig. 1 in this erratum. The thick colored curve shows the corrected estimated $h_{0}$, assuming an age of the black hole $(\mathrm{BH}) t_{\text {age }}=5 \times 10^{6} \mathrm{yr}$. The disfavored boson mass range presented in Sec. III A of this paper is hence updated to $6.4 \leq \mu /\left(10^{-13} \mathrm{eV}\right) \leq 8.0$, indicated by the dark

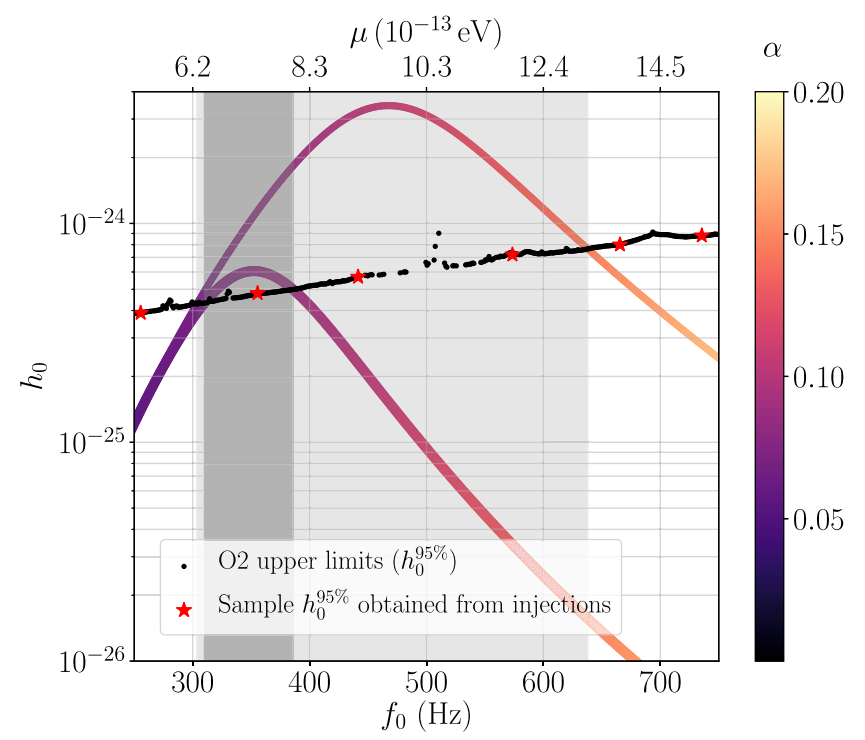

FIG. 1. Frequentist strain upper limits at $95 \%$ confidence $\left(h_{0}^{95 \%}\right)$ and disfavored scalar boson mass range. The colored curves show the numerically estimated signal strain $\left(h_{0}\right)$ as a function of boson mass (top axis) and GW frequency (bottom axis). The thick and thin curves correspond to $t_{\text {age }}=5 \times 10^{6} \mathrm{yr}$ and $1 \times 10^{5} \mathrm{yr}$, respectively. The color stands for the fine-structure constant $(\alpha)$. The black dots indicate $h_{0}^{95 \%}$ obtained from the search, assuming the electromagnetically measured orientation $l=27.1^{\circ} \pm 0.8^{\circ}$. The red stars mark $h_{0}^{95 \%}$ obtained through injections in $\mathrm{O} 2$ data in six sample 1-Hz sub-bands. Sub-bands without a marker were vetoed. The shaded region marks the parameter space where $h_{0}^{95 \%}$ beats the analytically estimated strain, and hence corresponds to the disfavored boson mass range without a detection: $6.4 \leq \mu /\left(10^{-13} \mathrm{eV}\right) \leq 8.0$ for $t_{\mathrm{age}}=5 \times 10^{6} \mathrm{yr}$ and $6.3 \leq \mu /\left(10^{-13} \mathrm{eV}\right) \leq 13.2$ for $t_{\mathrm{age}}=1 \times 10^{5} \mathrm{yr}$. The source parameters adopted in the analytic estimation are $M=14.8 M_{\odot}, \chi_{i}=0.99$, and $d=1.86 \mathrm{kpc}$.

\footnotetext{
*Corresponding author. ling.sun@anu.edu.au
} 
shaded region. This is a conservatively disfavored mass range [3], corresponding to a conservative choice of the $\mathrm{BH}$ age, $t_{\text {age }}=5 \times 10^{6} \mathrm{yr}$. We add an alternative choice of $t_{\text {age }}$ and the corresponding results in this erratum. If it is assumed that the $\mathrm{BH}$ is not much older than the x-ray binary jet, e.g., $t_{\text {age }}=10^{5} \mathrm{yr}$ [4], the estimated $h_{0}$ is significantly larger for $\mu \gtrsim 6.2 \times 10^{-13} \mathrm{eV}$ (thin colored curve), and a wider mass range of $6.3 \leq \mu /\left(10^{-13} \mathrm{eV}\right) \leq 13.2$ is disfavored (light shaded region).

The correction described in this erratum does not impact the results presented for the string axiverse scenario.

[1] M. Isi, L. Sun, R. Brito, and A. Melatos, Directed searches for gravitational waves from ultralight bosons, Phys. Rev. D 99, 084042 (2019).

[2] M. Isi, L. Sun, R. Brito, and A. Melatos, Erratum: Directed searches for gravitational waves from ultralight bosons [Phys. Rev. D 99, 084042 (2019)]; Phys. Rev. D 102, 049901 (2020).

[3] The authors thank Ilya Mandel for helpful input regarding the potentially younger age of the $\mathrm{BH}$.

[4] D. M. Russell, R. P. Fender, E. Gallo, and C. R. Kaiser, The jet-powered optical nebula of Cygnus X1, Mon. Not. R. Astron. Soc. 376, 1341 (2007). 